Highlights in basic autonomic neurosciences: changes to the autonomic nervous system associated with healthy ageing

lan J Edwards

Neuroscience, Physiology \& Pharmacology, University College London, London, UK DOI: 10.1016/j.autneu.2014.04.004

With a worldwide ageing population, it is of vital importance to understand how the autonomic nervous system adapts as we age to meet the ever changing demands on the body. Ageing is associated with changes to the cardiovascular system (including a steady increase in blood pressure), a variety of gastrointestinal disorders and urinary incontinence, as well as many other important system changes which are under autonomic control. The cardiovascular changes associated with healthy ageing are further complicated in women by the change in sex hormone levels associated with the menopause: ultimately the loss of the cardio-protective actions of oestrogen as highlighted in the first article below.

Barnes JN, Hart EC, Curry TB, Nicholson WT, Eisenach JH, Wallin BG, Charkoudian $\mathrm{N}$, Joyner MJ. Ageing enhances autonomic support of blood pressure in women. Hypertension. 2014 63(2):303-8.

Article summary

Ageing is associated with concurrent increases in sympathetic nerve activity and arterial blood pressure in both sexes. In women, post-menopause, the relationship between sympathetic nerve activity and total peripheral resistance becomes much stronger - indicative of a difference in how women regulate their blood pressure preand post-menopause. The authors examined the effect of ganglionic blockade in the correlation between muscle sympathetic nerve activity and arterial blood pressure in pre- and post-menopausal women. Muscle sympathetic nerve activity (MSNA) was approximately 2 fold higher in post-menopausal women at baseline than in the younger women, and was associated with significantly higher systolic blood pressure and plasma noradrenaline. Ganglionic blockade using trimethaphan caused a significant reduction in mean, systolic and diastolic blood pressure in the postmenopausal women, but did not cause any significant alterations in the younger women.

\title{
Commentary
}

This paper beautifully highlights the shift in the importance of the sympathetic nervous system in the regulation of blood pressure in normotensive women as they pass from pre- to post-menopausal. Young women have very low levels of MSNA, lower than age matched males and post-menopausal women. The low levels of MSNA in young women are associated with low levels of circulating noradrenaline 
and low total peripheral resistance. Post-menopause there is an obvious shift to higher MSNA and circulating noradrenaline, and an increase in total peripheral resistance. At this postmenopausal stage the correlation between MSNA and total peripheral resistance becomes much tighter, and more reminiscent of the relationship between the two observed in men. The most interesting finding here is how little contribution the sympathetic nervous system appears to have in the maintenance of blood pressure in young women.

Much of the increased potency of the sympathetic nervous system post-menopause can be attributed to changes in the levels of sex hormones. In rats ovariectomy increases $\alpha$-adrenoceptor mediated vasoconstriction (Berezan et al. 2008) and decreases the sensitivity of $\beta$-adrenoceptors (Ferrer et al. 1996) reducing their vasodilatory capacity. Taken together with the increase in circulating noradrenaline which accompanies the increase in MSNA observed post-menopause, there is an obvious mechanism underlying the shift in the contribution of the sympathetic nervous system to the regulation of blood pressure in post-menopausal women. This shift towards a sympathetically driven cardiovascular system may be one of the most important driving factors behind the increase in hypertension in women postmenopause.

Johnson TL, Tulis DA, Keeler BE, Virag JA, Lust RM, Clemens S. The dopamine D3 receptor knockout mouse mimics ageing-related changes in autonomic function and cardiac fibrosis. PLoS One 2013 8(8) e74116

\section{Article summary}

As mentioned previously; blood pressure steadily increases with age. It has also been noted that there is an age related decline in the function of the dopamine D3 receptor which has been implicated in the development of hypertension. This study compared the progression of hypertension in wild type and D3KO mice. In wild type mice blood pressure values remain steady for the first year and then increase dramatically in the mouse's second year, however the D3KO mice reached this elevated pressure level by the age of 2 months. The increases in blood pressure were also accompanied by bradycardia in both the 2 year old wild type and 2 month old D3KO mice. Clear morphological changes in the structure of the heart were evident in the aged wild type mice, but these were not mimicked by the D3KO mice suggesting that the hypertension observed in these animals was not due to anatomical changes or cardiac hypertrophy. Both aged and D3KO mice showed a significant, and comparable, reduction in both ejection fraction and fractional shortening. Both the aged and D3KO mice also showed clear evidence of cardiac fibrosis and displayed increased levels of collagen and increased activity of matrix metalloproteinase 9 .

Commentary 
This paper shows that some of the hallmarks of a healthy ageing heart are shared by mice lacking the dopamine D3 receptor. Dysfunction of the D3 receptor has previously been linked to the development of hypertension, and this receptor is expressed in the left and right ventricles of the heart and also in the proximal tubules of the kidney. D3KO mice develop hypertension comparable to that seen in 2 year old mice with in the first 2 months of life, which is associated with increased in collagen levels and left ventricular fibrosis that is again comparable to that of the 2 year old wild type mice. In rodents, and indeed in humans, it has been demonstrated that ageing is associated with a loss of ventricular myocytes, which is often associated with the deposition of interstitial collagen and fibrosis. It is therefore very interesting that the D3KO mice can mimic this condition of the aged heart in such a short amount of time. It is also interesting that so many of the reported measures were similar between the 2 month old D3KO mice and the 2 year old wild type mice, with the exception of the morphological changes - which presumably take a little longer to develop than 2 months.

Callegaro CC, Taylor JA. Age-related effects of vagotonic atropine on cardiovagal baroreflex gain. Neurobiology of ageing 2012 33(2): 368-74

\section{Article summary}

Advancing age is associated with a decrease in the sensitivity of the cardiac baroreflex, which could potentially be caused through stiffening of the barosensory vessels or a reduced vagal outflow or a combination of the two. This study used low doses of atropine to enhance the central components of the baroreflex arc. As atropine has no effect upon the firing of baroreceptive afferents this allows the authors to better determine where in the reflex arc the decrease in sensitivity arises. The authors demonstrated that low doses of atropine produced bradycardia in both young and old individuals, but only increased baroreflex gain in the older subjects. The increase in baroreceptor gain in the older individuals should not have occurred through an increase in the sensitivity of the baroreceptive afferents as these are unaffected by atropine, rather it is likely that it is through increasing the activity in vagal efferents.

\section{Commentary}

This article nicely pushes the focus of the loss of cardiac baroreflex gain onto the central and efferent components of the baroreflex arc. The low doses of atropine used here are parasympathomimetic, evident by the pronounced bradycardia observed in both the young and old experimental subjects - unlike the response to higher doses which have a strong parasympatholytic effect and produce tachycardia. Compared to the younger subjects the elderly volunteers had obvious deficits in baroreflex gain, which could be traced back to both the neural and mechanical components. Upon the administration of atropine these elderly subjects displayed an enhanced baroreflex sensitivity with a significant improvement of the neural 
component of the reflex - a response that was not observed in the younger subjects. Further, subjects with the lowest baroreflex sensitivity showed the greatest increase in baroreflex gain upon administration of atropine. The authors propose that this increase in baroreflex gain is likely to be through increased activation of vagal efferents, either directly or through an enhancement of neurotransmission within the nucleus of the solitary tract. It has been shown that aged rats have a reduced number of synapses within the cardiac ganglion (Ai et al. 2007), as well as a decrease in the number of muscarinic receptors in the sinoatrial node in humans. This obvious deficit in the vagal innervation of the heart could easily account for the decline in baroreflex sensitivity, which could also be reduced by increasing the activation of the existing pool of cardiac vagal efferents. As the elderly are more prone to hypertension, as discussed in the previous articles, maintenance of vagal tone to prevent surges in blood pressure is of critical importance. It is therefore very encourageing to see that the deficit in baroreflex function can be restored through increasing vagal tone using low doses of atropine.

Phillips RJ, Pairitz JC, Powley TL. Age-related neuronal loss in the submucosal plexus of the colon of Fischer 344 rats. Neurobiology of Ageing 2007 28(7): 1124-37

Article summary

Faecal incontinence, chronic constipation and inflammatory bowel disease are all common colonic disorders amongst the elderly. Colonic function is controlled via autonomic innervation of the myenteric and submucosal plexuses, and therefore it is important to understand how this innervation changes over the course of normal ageing. This article shows that there is a progressive reduction in the number of neurones per ganglia in the myenteric plexus in both the proximal and distal colon. This reduction in submucosal neurone number is also associated with extensive axonopathy of sympathetic efferents innervating the submucosal ganglia.

\section{Commentary}

The neuronal loss reported here within the submucosal plexus follows the same pattern as that observed in the myenteric plexus. For both plexuses there is an oral to anal gradient in the severity of the neuronal loss which begins at approximately 12 months and then progresses in a linear manner. Therefore, over the course of normal ageing there is a progressive loss of neuronal control of the bowel which may contribute to the prevalence of colonic disorders experienced by the elderly. Further to this it is evident that there is a significant disruption in the sympathetic innervation of the colon, evident by the marked axonopathies observed in tyrosine hydroxylase immunoreactive fibres innervating the submucosal plexus. As the sympathetic nervous system acts to decrease gut motility, this loss of sympathetic control of the bowel may contribute to faecal incontinence. Again these dystrophic sympathetic fibres have also been reported within the myenteric plexus. A recent review in Autonomic Neuroscience: Basic and Clinical by the same authors also revealed that 
vagal afferent innervation of the GIT showed pronounced dystrophy and regressive morphological changes (Phillips et al. 2010). It is clear that the aged colon has major deficiencies in both its intrinsic and extrinsic innervation which could very easily contribute to the loss of function that is observed in the elderly population, but a more complete understanding of how these deficits of innervation manifest is required.

Zhao W, Aboushwareb T, Turner C, Mathis C, Bennett C, Sonntag WE, Andersson $\mathrm{KE}$, Christ G. Impaired bladder function in ageing male rats. Journal of Urology 2010 184(1): 378-85

\section{Article summary}

The prevalence of problems with bladder dysfunction increases with age, and as such poses more of a problem with our ageing population. This study used aged rats to investigate how bladder function changes as part of the ageing process. Strips of detrusor muscle from aged bladders showed a significant reduction in contractility in response to carbachol and electrical field stimulation. Histological examination revealed urothelial thinning, decreased muscle mass and an increase in the deposition of collagen in the aged bladder. In vivo analysis of bladder function showed that the aged bladder had an increased capacity, with a larger residual volume and micturition volume accompanied by a significant increase in the micturition frequency.

\section{Commentary}

This study stood out as it combines in vivo cystometric data and in vitro contractility data and histology. All three parts of this study displayed striking similarity to the ageing human bladder. The aged human bladder is often overactive but hypocontractile, with many voidings and a high residual volume, and is often associated with fibrosis and the deposition of collagen. Another interesting point of this study was that electrical field stimulation in the presence of atropine revealed a non-muscarinic, presumably ATP mediated, contractile component which comprised a larger proportion of the total contractile profile in the aged bladder compared to the young. Although the majority of overactive bladder treatments are based around muscarinic receptor antagonists there is evidence to suggest that the ATP mediated contractile component is increased in aged patients.

\section{Conclusion}

The ageing autonomic nervous system undergoes many anatomical and functional changes which greatly reduce its ability to control its target organs. A common feature that seems to accompany ageing of the autonomic nervous system is a reduction in the innervation of its target organs. This is clearly evident for the heart in the reduction in baroreflex sensitivity and in the colon, where there is extensive loss 
of both intrinsic and extrinsic neurones. This loss of innervation is often accompanied by collagen deposits within the target organ reducing the functionality of the organ even further.

\section{References}

Ai, J., Gozal, D., Li, L., Wead, W.B., Chapleau, M.W., Wurster, R., Yang, B., Li, H., Liu, R., \& Cheng, Z. 2007. Degeneration of vagal efferent axons and terminals in cardiac ganglia of aged rats. J.Comp Neurol., 504, (1) 74-88 available from: PM:17614301

Barnes, J.N., Hart, E.C., Curry, T.B., Nicholson, W.T., Eisenach, J.H., Wallin, B.G., Charkoudian, N., \& Joyner, M.J. 2014. Ageing enhances autonomic support of blood pressure in women. Hypertension, 63, (2) 303-308 available from: PM:24324040

Berezan, D.J., Xu, Y., Falck, J.R., Kundu, A.P., \& Davidge, S.T. 2008. Ovariectomy, but not estrogen deficiency, increases CYP4A modulation of alpha(1)-adrenergic vasoconstriction in ageing female rats. Am.J.Hypertens., 21, (6) 685-690 available from: PM:18437124

Callegaro, C.C. \& Taylor, J.A. 2012. Age-related effects of vagotonic atropine on cardiovagal baroreflex gain. Neurobiol.Ageing, 33, (2) 368-374 available from: PM:20541842

Ferrer, M., Meyer, M., \& Osol, G. 1996. Estrogen replacement increases betaadrenoceptor-mediated relaxation of rat mesenteric arteries. J.Vasc.Res., 33, (2) 124-131 available from: PM:8630345

Johnson, T.L., Tulis, D.A., Keeler, B.E., Virag, J.A., Lust, R.M., \& Clemens, S. 2013. The dopamine D3 receptor knockout mouse mimics ageing-related changes in autonomic function and cardiac fibrosis. PLoS.One., 8, (8) e74116 available from: PM:24023697

Phillips, R.J., Pairitz, J.C., \& Powley, T.L. 2007. Age-related neuronal loss in the submucosal plexus of the colon of Fischer 344 rats. Neurobiol.Ageing, 28, (7) 11241137 available from: PM:16793176

Phillips, R.J., Walter, G.C., \& Powley, T.L. 2010. Age-related changes in vagal afferents innervating the gastrointestinal tract. Auton.Neurosci., 153, (1-2) 90-98 available from: PM:19665435

Zhao, W., Aboushwareb, T., Turner, C., Mathis, C., Bennett, C., Sonntag, W.E., Andersson, K.E., \& Christ, G. 2010. Impaired bladder function in ageing male rats. J.Urol., 184, (1) 378-385 available from: PM:20488483 